Original article

\title{
Ambivalence in distinguishing double burden of malnutrition among school children in three districts of south India
}

\author{
Chandana Hombaiah $^{\mathrm{a}}$, Anil S. Bilimale ${ }^{\mathrm{a}, *}$, B. Madhu ${ }^{\mathrm{a}}$, M.R. Narayana Murthy ${ }^{\mathrm{b}}$ \\ ${ }^{a}$ Department of Community Medicine, JSS Medical College, JSS AHER, Mysuru, Karnataka, 570015, India \\ ${ }^{\mathrm{b}}$ Department of Community Medicine and School of Public Health, JSS Medical College, JSS AHER, Mysuru, Karnataka, 570015, India
}

\section{A R T I C L E I N F O}

\section{Keywords:}

Dual burden

Growth chart

Adolescent nutrition

Obesity

Body mass index (BMI)

\begin{abstract}
A B S T R A C T
Objectives: To assess the nutritional status of school-going children in the age group 6-17 years and to compare BMI growth percentile charts of WHO and IAP (Indian Academy of Paediatrics).

Methods: A cross-sectional study was conducted among school children aged 6-17 years studying in 44 schools of three districts in South India, between 2018 and 2019. A total of 8688 students were assessed for their height and weight. Anthropometric data were plotted on both WHO and IAP BMI charts.

Results: There were $50.1 \%$ boys and $49.9 \%$ girls; $32.1 \%$ were urban and $67.9 \%$ from rural localities. According to the WHO BMI chart, $14.7 \%$ of the school students were severely thin, $15.8 \%$ were thin, $6.9 \%$ were overweight and $2.2 \%$ were obese. Under-nutrition among boys was $9.7 \%$ more prevalent than girls. Overweight prevalence was higher in girls $(9.6 \%) ; 4.1 \%$ higher prevalence among urban than rural girls. The difference between the two BMI chart measurements for $<3$ rd percentile possessed a variation of $24.2 \%$ for boys and $12.3 \%$ for girls. Significant undernutrition (53.6\%) was among 6-8yrs and overnutrition (11\%) among adolescents.

Conclusion: The study reveals a double burden of malnutrition with gender, age, and geographic disparity. Boys were severely thin than girls while girls were overweight than boys. Rural students were underweight whereas urban students were overweight and obese. Thinness was inversely proportional to age. The sensitivity to determine under-nutrition was with the WHO chart whilst the IAP chart was for over-nutrition.
\end{abstract}

\section{Introduction}

Childhood is a critical period for a person's overall growth and development throughout the lifespan. ${ }^{1}$ School-going age is a formative period that transforms the child into a successful adult, both physically and mentally. ${ }^{3}$ Nutrition of school-going age group and adolescents play a major role in the prevention of maternal and infant mortality through a life cycle approach. ${ }^{4}$

The 5-19-year-old children constitute $27 \%$ of the total population in India. They form a major population in urban, semi-urban, and rural districts across the nation. There are only a few nationally representative surveys available on the nutritional status of boys and girls aged 5-19 years. Few of the studies conducted with a smaller sample size indicate the incidence of nutrient deprivation and its effects are high in children between the ages of 5 and 15 years. $^{2}$ This age group has been seriously challenged by the transition of nutritional problems from under-nutrition to double burden of malnutrition. This means the co-existence of both under-nutrition and over-nutrition which is distributed in all types of demography. Earlier, it was understood that under-nutrition and over-nutrition were separate problems. However, nowadays the scientific community realizes that these aspects are inter-connected. ${ }^{5}$ Many low- and middle-income countries resume co-existing with issues of undernutrition and infectious diseases. Also, they are encountering a sharp increase in non-communicable disease risk factors such as obesity and overweight, especially in urban settings. ${ }^{6}$ Therefore, monitoring these aspects becomes important to understand causation and effect.

Growth monitoring is a multilevel strategy that is applied to ascertain early growth retardation, encourage optimum growth, generate awareness about growth among mothers, strengthen the delivery of primary health care, and recognize those at risk of malnutrition. ${ }^{7}$ One such way of implementing this monitoring strategy is by conducting a thorough evaluation of children in specific growth and nutrition domains, and plotting the finding by weight, height, or Body Mass Index

\footnotetext{
* Corresponding author.

E-mail addresses: Chandana.h8109@gmail.com (C. Hombaiah), anilbilimale@gmail.com (A.S. Bilimale), drmadhusri@gmail.com (B. Madhu), drmrnmurthy@ yahoo.co.in (M.R. Narayana Murthy).
} 
(BMI) in the growth charts. ${ }^{7}$ The growth charts are then used to evaluate the growth and development of the children. In India, policymakers refer to the World Health Organization (WHO) growth chart, ${ }^{8}$ as well as an Indian Academy of Paediatrics (IAP) growth chart. ${ }^{9}$ The IAP growth chart is specifically developed for Indian children. The difference between IAP and WHO growth charts and the choice of the chart affects the magnitude of nutritional status. ${ }^{10}$ Therefore, it is important to assess whether using the growth references of the WHO will result in changes in the estimated prevalence of malnutrition compared to the growth charts of the IAP and, if so, then index the degree of change. ${ }^{10}$ During formative years it would be prudent to determine these growth monitoring strategies, especially so as a myriad of socio-economic factors can affect the implementation.

It would be interesting to see if the aforementioned factors and behavior are observed in a larger cohort while determining the applicability of growth monitoring tools for the varied nutritional states. The following are the objectives of the study:

1. To assess the nutritional status of school-going children in the age group 6-17 years

2. To compare BMI growth percentile charts of WHO and IAP

\section{Methods}

A cross-sectional study was conducted across 44 schools of Mysuru, Chamarajanagar, and Mandya districts in the State of Karnataka, India between September 2018 and March 2019. This comprised of government-aided schools from both urban and rural areas under the administration of educational institutions in the region.

Among the total 9110 students enlisted, 422 of them were absent on the day of the examination, and thus 8688 students were available for the school health program. Data collection was done in person by the post-graduate students from the medical college. Students between the ages of 6-17 years who were present on the day of examination were included in the study. In India, school enrollment starts from $5 \mathrm{y} 10 \mathrm{~m}$ onwards, and hence our participants were mainly above 6 years.

A health screening was conducted to assess the height and weight of the children. The weight of the child was recorded in kilograms using an analog weighing scale; height was labeled in centimeters on the wall with the support of measuring tape. Children assembled to stand bare feet with heels, back and occiput facing the wall with the Frankfurt plane being parallel to the ground. A scale was placed at the topmost point of the head and reading was taken avoiding parallax. The readings were taken by the qualified doctors who were trained before the study and the readings were supervised. Throughout the study period, the same instrument was used. Anthropometric data were plotted individually on both WHO and IAP charts. For the comprehensive evaluation, the nutritional status, Body Mass Index (BMI) was calculated manually utilizing the formula weight $(\mathrm{Kg}) /[$ height $(\mathrm{mt})] .{ }^{2}$ Using the $\mathrm{z}$-scores chart from WHO, five categories were identified. A BMI of below -3SD was labeled as severe thinness, below -2SD as thinness, below $+1 \mathrm{SD}$ as normal, below $+2 \mathrm{SD}$ as overweight, and above $+2 \mathrm{SD}$ as obesity. ${ }^{8}$

\subsection{Ethical aspects}

The study was approved by the institutional ethical committee (JSSMC/IEC/2509/Aca Study 10/2018-19) and permission from respective educational authorities was obtained. Assent from the parents was taken by the educational authorities for the examination of children.

\subsection{Statistics}

The data obtained was coded and entered into Microsoft Excel worksheet 2016 and was later imported and analyzed using SPSS version 23 (licensed to JSS AHER). Descriptive statistics like frequencies were applied for the variables like age, gender, locality, and BMI status of students. Data were represented as graphs as applicable, and it was imported from Microsoft Powerpoint. A chi-square test was applied to find out the association of malnutrition with age, gender, and locality.

\section{Results}

A total of 8688 students were examined, 4345 (50.1\%) were boys and 4343 (49.9\%) were girls. Of these, 2783 students (32.1\%) belonged to urban localities and 5905 students (67.9\%) hailed from rural areas (Fig. 1). There were 1467 (52.7\%) urban boys, 1316 (47.3\%) urban girls, 2878 (48.7\%) rural boys, and 3027 (51.3\%) rural girls (Fig. 1). A chisquare test was applied to find the association of BMI status with locality and gender. Both were found to be statistically significant at a pvalue of $<0.001$ (Fig. 2).

The BMI status was different among the age groups (6-8yrs, 9-11yrs, 12-14 yrs and 15-17yrs). Among the children between the age group of 6-8 years, $40.2 \%$ belonged to the severe thinness category, and among the $12-14$ years age category, $8.5 \%$ were overweight. This was found to be statistically significant at a p-value of $<0.001$ (Fig. 3).

A comparison of BMI growth percentile charts between $\mathrm{WHO}^{9}$ and $\mathrm{IAP}^{10}$ was done. It was noted that $39.6 \%$ of the boys under the WHO BMI percentile growth chart were shown to have $<3$ rd percentile growth while IAP shows $15.4 \%$ of the boys have $<3$ rd percentile of growth, respectively. While $26.5 \%$ of the girls have $<3$ rd percentile growth according to the WHO BMI percentile growth chart whereas IAP shows $14.2 \%$ of the girls have $<3$ rd percentile growth, respectively (Fig. 4).

\section{Discussion}

The study assessed the school students aged between 6 and 17 years for their nutritional status and general morbidity profile. The results revealed that $14.7 \%$ of the school students were severely thin, $15.8 \%$ of students were thin, $6.9 \%$ were overweight and $2.2 \%$ of students were obese. The current study shows that boys were severely thin (18\%) when compared to girls (11.4\%) and girls (7.6\%) were more overweight when compared to boys (6.3\%). Comparison between rural and urban students

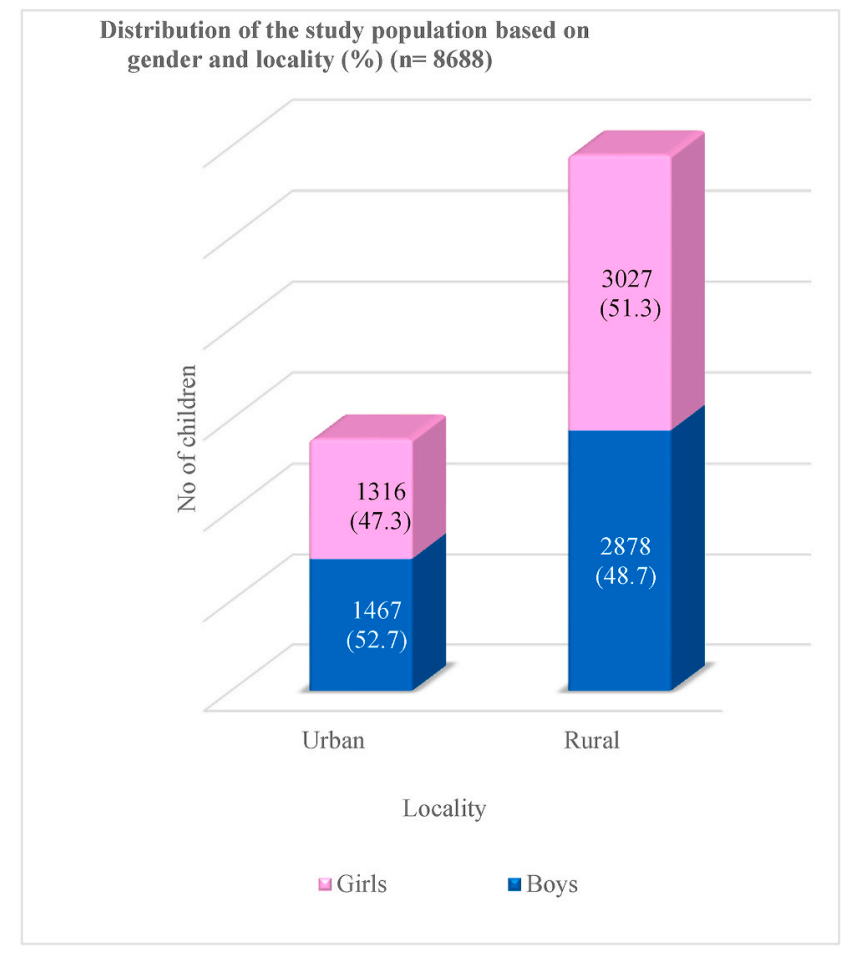

Fig. 1. Distribution of the study population based on gender and locality (\%) $(\mathrm{n}=8688)$. 


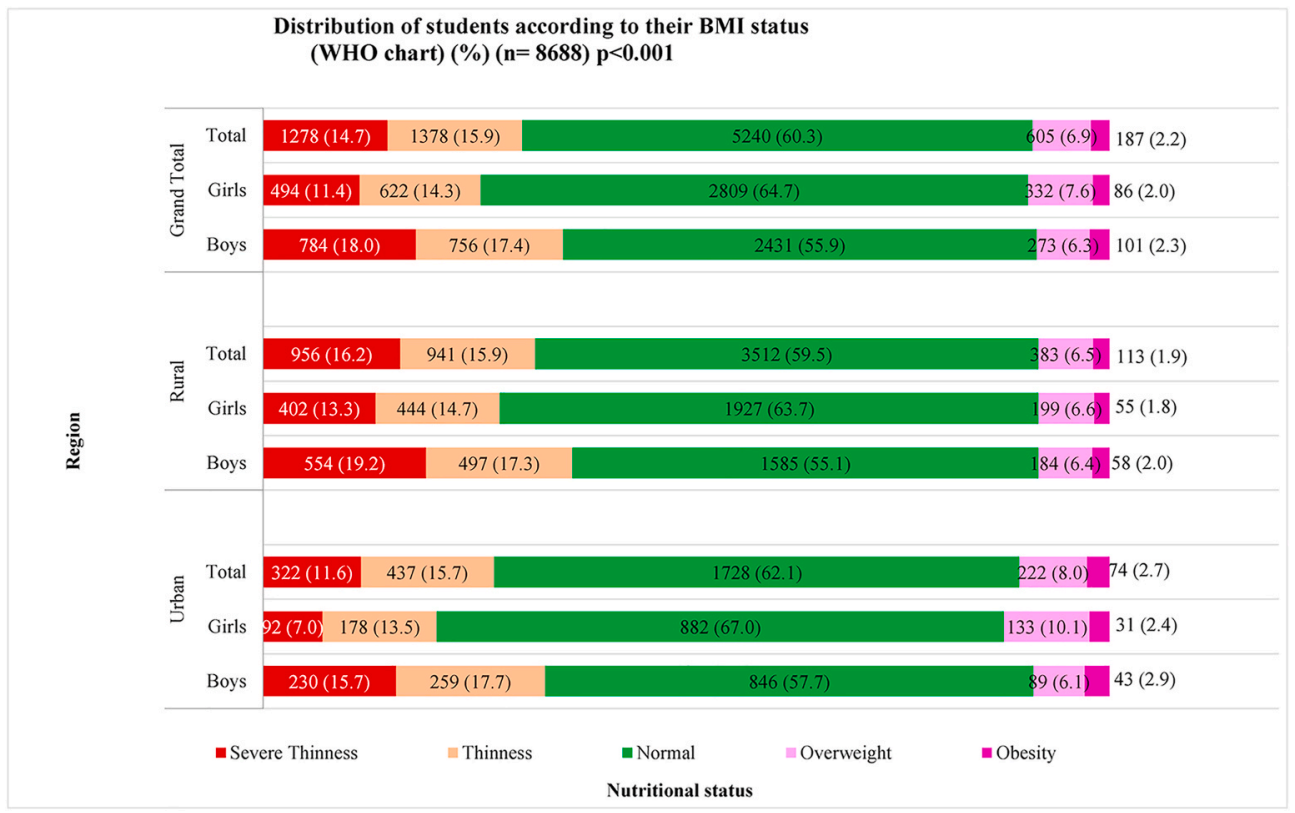

Fig. 2. Distribution of student according to their BMI status (WHO chart) $(\%)(\mathrm{n}=8688) \mathrm{p}<0.001$.

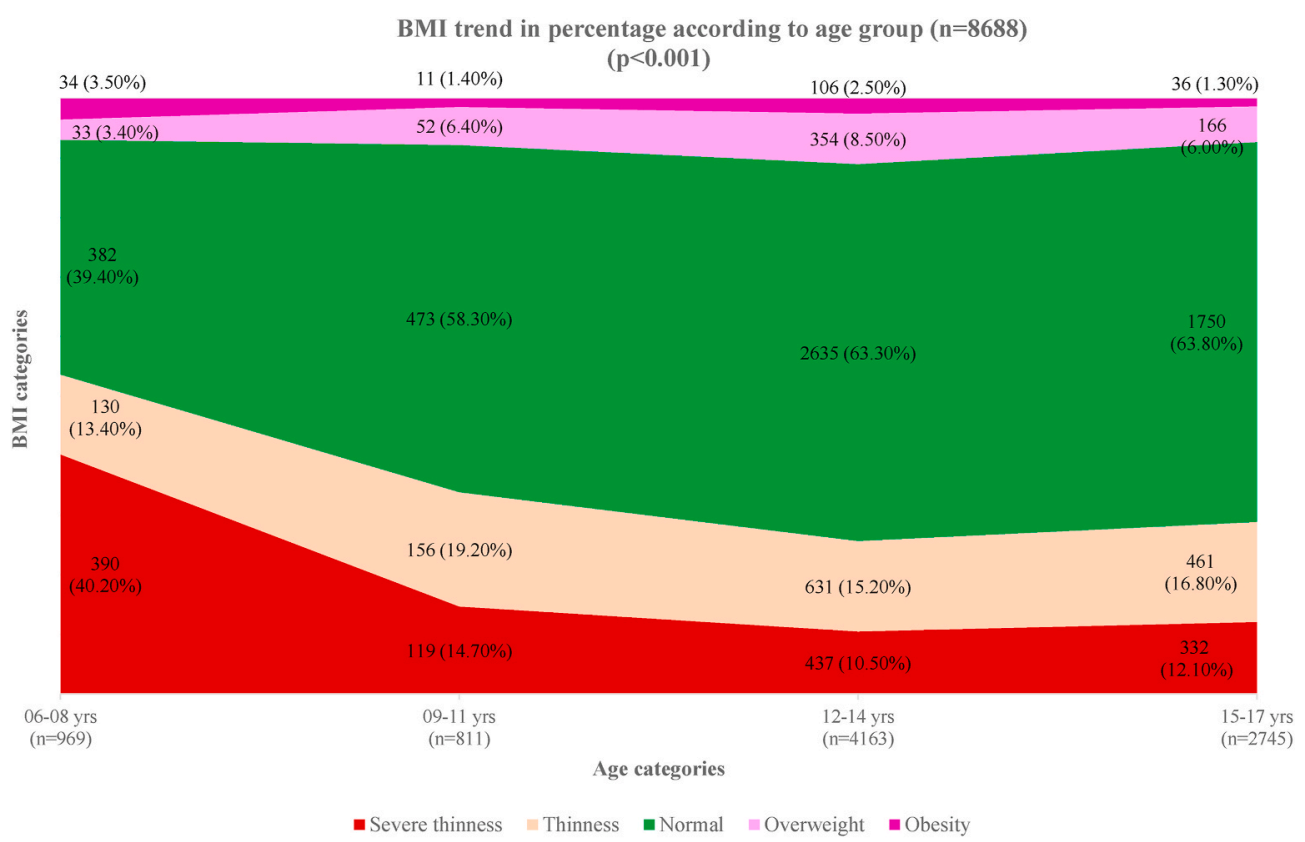

Fig. 3. BMI trend in percentage according to age group $(n=8688)$.

revealed that more of the rural students $(16.2 \%)$ were severely thin and more urban students were overweight $(8 \%)$ and obese $(2.7 \%)$, respectively. Severe thinness was maximum in the age group of 6-8 years $(40.2 \%)$ which has a decreasing trend as age increased (15-17yrs $12.1 \%)$. Overweight was maximum in the $12-14$ yrs age group $(8.5 \%)$.

Fewer studies have been conducted in India, regarding the nutritional status of children above six years of age. A study conducted in the southern part of India (Hasan I, 2010) ${ }^{11}$ showed that $68 \%$ of students were underweight, while the current study showed a lesser prevalence (both rural and urban 30.5\%) and still lesser (27.3\%) in urban students. The study was done among the government school children in urban areas belonging to poor socio-economic status while the current study was done among the government-aided schools in both rural and urban areas. In a study conducted in another south Indian site, two BMI charts were used. According to CDC (Centre for disease control) chart more children $(40.8 \%)$ were underweight/thin when compared with the current study (30.5\%) and when compared with the Agarwal chart, lesser prevalence $(24 \%)$ of underweight/thinness was noticed in their study. ${ }^{3}$ The studies conducted by Ranu Rawat et al. and JP Singh et al. in other parts of India, showed a higher prevalence of under-nutrition of $48 \%$ and $41 \%$, respectively among the school students aged above 6 years when compared to the current study. ${ }^{12,13}$ The inference is that under-nutrition is still a significant problem, although a reduction in the prevalence is observed in an urban area. Improvement is observed in recent years. North Indian states have a higher prevalence compared to South Indian states. Besides, variation is seen when different types of charts are used.

The current study reveals that overnutrition is $9.2 \%$ (Obesity $-2.2 \%$ 


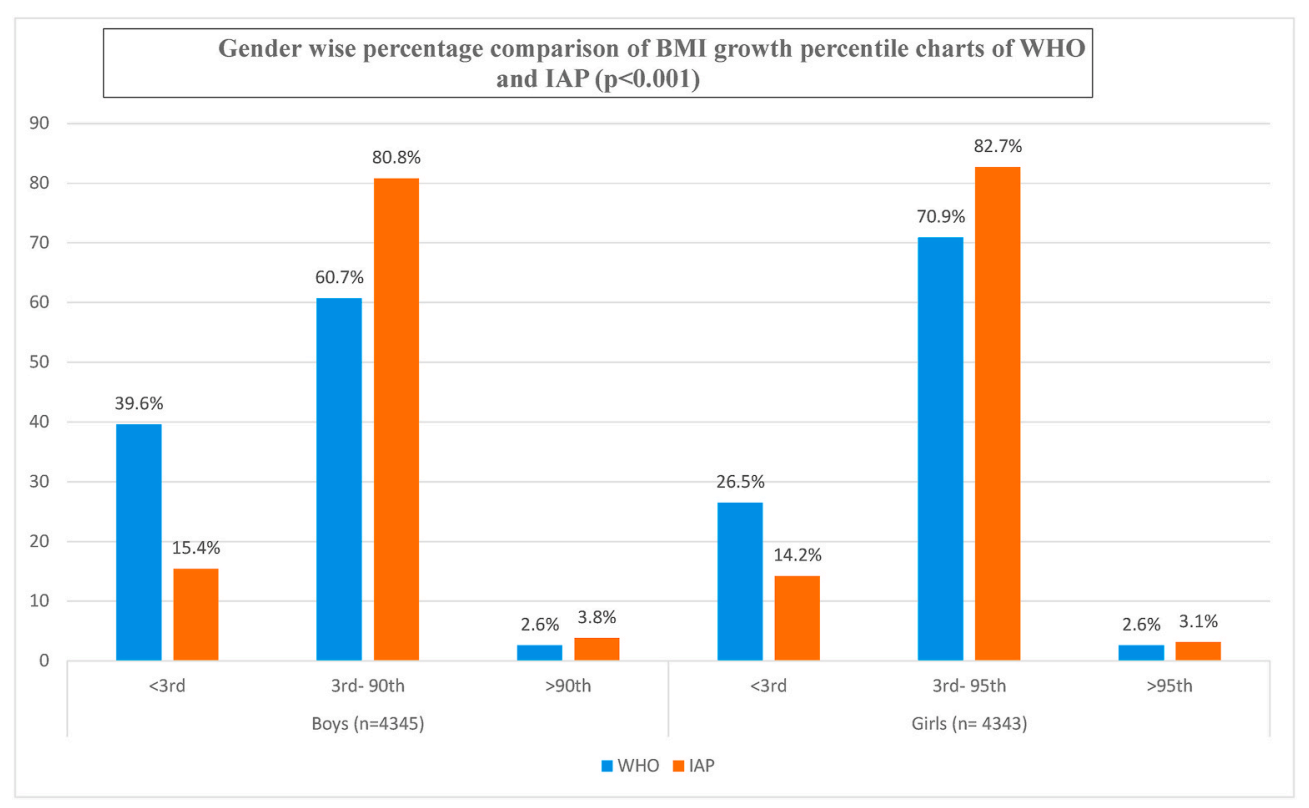

Fig. 4. Gender wise percentage comparsion of BMI growth percentile chart of WHO and $\operatorname{IAP}(\mathrm{P}<0.001)$.

and Overweight $-6.9 \%$ ). A systematic review done by Harish Ranjani et al. revealed that in children above 5 years of age, the prevalence of obesity varied between 2 and $8 \%$. The overweight rates in northern and eastern India were about two times higher than in southern India. Among the higher socio-economic groups, overweight and obese rates are rising for children and adolescents, but among the lower socioeconomic group underweight is still a significant concern. ${ }^{14}$ Globally girls have $>5 \%$ and boys $>8 \%$ prevalence of over-nutrition as in 2016 . ${ }^{15}$

Dual burden: In the present study, we can see the co-existence of both undernutrition and overnutrition, which is known as the "double burden of malnutrition". ${ }^{16}$ The prevalence of double burden in the current study is around $39.7 \%$ with more prevalence among boys (44.1\%) than girls (35.3\%). Also, this is higher in the 6-8yrs age group $(60.6 \%)$. A study done in Mauritius opined that the co-existence of undernutrition and overnutrition among the Indian ethnic children is because of the socio-economic groups, as some have insufficient resources, and some have more than enough resources. ${ }^{17}$ In a study conducted by Doak C M, it was observed that household double burden prevalence in Kyrgyzstan was $12.6 \%$, Indonesia $11.2 \%$, and Brazil $8.2 \%$, respectively. ${ }^{18} \mathrm{~A}$ study by Popkin mentions the double burden of malnutrition (DBM) is especially prevalent in sub-Saharan Africa, East Asia, South Asia, and the Pacific. India along with China are the top consumers of sugary beverage manufacturers. ${ }^{5}$ Further, the low and middle-income countries have been transitioning towards the increasing burden of DBM which is due to the change in the food system, sedentary lifestyle, and economic change. Additionally, urbanization has brought a change in the work style which has led to increased consumption of processed foods, ready to heat, and ready to eat foods.

The current study shows that boys (18\%) were severely thin when compared to girls (11.4\%). Similar disparities have been mirrored in the studies conducted by Ranu Rawat et al. JP Singh et al. and Izhar Hasan. It revealed that underweight prevalence for boys was $56 \%, 44.56 \%$, and $57.94 \%$ respectively, while for girls was $44 \%, 37.32 \%$, and $42.06 \%$ respectively. ${ }^{11-13}$ In the present study, girls (7.6\%) were found to be more overweight than boys $(6.3 \%)$ both in urban and rural areas. Understandably, the urban dwellings and lifestyles have led to poor outdoor physical activity with an increased amount of time being spent on watching television/mobile, passive in household activities, having dinner as the heaviest meal of the day, and having less healthy nutritious foods. $^{19}$

In this study, students studying in rural areas were more underweight than the urban areas and students studying in urban areas were more overweight and obese than the rural areas. This could be because of a higher prevalence of poverty, poor education of parents, lack of hygiene, and defecation within premises in the rural areas. Also, the consumption of locally available grains and vegetables in rural areas may lead to lesser availability of a balanced diet especially concerning vitamins and minerals. ${ }^{20}$ Whereas, urbanization will lead to positive adjustments in children's diets, as well as a variety of unhealthful dietary changes like increased consumption of processed foods, sugars, salt, and overconsumption of saturated and trans fats. ${ }^{16}$ The digital screen time, unavailability of playgrounds, decreased socialization, private tuition time have been increasing due to urbanization and this can also be a factor for lesser physical activity.

Age-related variations: The current study reveals that as age increases under-nutrition decreases $(53.6 \%$ in $6-8 \mathrm{yrs}$ and $28.9 \%$ in $15-17 y r s)$ and overweight increases $(6.9 \%$ in $6-8 y r s$ and $11 \%$ in $12-14 y r s)$. In a study by Galloway R, the prevalence of undernutrition among late adolescents (15-19 yrs of age) was 47\% among girls and $58 \%$ among boys in India (2005-06). It was as low as 1\% in Egypt, $5 \%$ in Equatorial Guinea, and as high as $66 \%$ in Ethiopia. The prevalence of over-nutrition is $10 \%$ in 13 countries. In 2005-2006, the over-nutrition prevalence in India was $2 \%$, and as high as $43 \%$ in Egypt (2014). ${ }^{2}$ In most of the Eastern Mediterranean, Western Pacific, and American regions it is $>25 \%$ among adolescents. Whereas the current study has measured the BMI of late adolescents between 15 and $17 \mathrm{yrs}$ of age with a prevalence of under-nutrition being $28.9 \%$ and over-nutrition being $7.3 \%$. Although the under-nutrition in India has reduced by almost $45 \%$, Overnutrition has increased more than three times in 13 years. ${ }^{2}$ Over-weight and obesity in adolescence are associated with chronic disease and mortality in later life. ${ }^{15}$

Measuring tool ambivalence: We compared growth centile charts between WHO and IAP for school students aged 6-17 years. Measurements done using the WHO chart for boys showed that $39.6 \%, 60.7 \%$, and $2.6 \%$ belong to $<3 \mathrm{rd}$, normal, and $>90$ th percentile growth, respectively. Whereas, according to IAP among the boys $15.4 \%, 80.8 \%$, and $3.8 \%$ belong to $<3 \mathrm{rd}$, normal, and $>90$ th percentile growth, respectively. Among the boys, it is noticed that the difference between WHO and IAP, in $<3$ rd percentile is equal to $24.2 \%$ and for $>90$ th percentile it is $1.2 \%$, and also WHO categorizes more boys under $<3$ rd percentile growth while IAP categorizes more boys towards $>90$ th percentile growth. Among the girls, according to the WHO chart $26.5 \%$, 
$70.9 \%$, and $2.6 \%$ belong to $<3$ rd, normal, and $>95$ th percentile growth; but according to the IAP chart $14.2,82.7 \%$, and $3.1 \%$ belong to $<3$ rd, normal and $>95$ th percentile growth. The difference between WHO and IAP charts for girls in the $<3$ rd percentile is $12.3 \%$ and for $>95$ th percentile is $0.5 \%$. The difference between WHO and IAP charts is greater in the boys' charts than in the girls' charts. A similar kind of study was done in Suratkal, ${ }^{3}$ where they compared CDC and Agarwal charts. Different values may create confusion among pediatricians, and they may influence national statistics and decision-making. When Indian rural population distribution was more during 1990 (75\%), underweight was a major concern and we required a sensitive tool to identify it. When we are in the urbanization era, a sensitive tool to identify overweight is required. Since the WHO tool was developed from multi-centric studies around the world (different races), it was required and relevant when we were not able to create a tool for our country. Hence, multi-centric studies at various places in India are required to decide on a single pertinent tool to be used for Indian children. ${ }^{21}$ WHO stated that it would not be possible to have prescriptive growth standards for the 5-18yrs age group because environmental variables in this age group cannot be controlled. Hence charts by the WHO for 5-18-year-old children are based on the statistical remodeling of the 1977 National Centre for Health Statistics data and are called growth references and not standards. Hence, it is inevitable to have country-specific growth charts to monitor the growth of children between 5 and 18 years. WHO has recommended updating the growth references every decade. ${ }^{22}$

The limitation of our study is that the selection of schools was from the schools governed by the same organization and was confined to South Karnataka. The socio-economic status of the children's families was not assessed which plays a major role in the nutritional status.

The strength of our study is: i) Large sample size with enumeration technique ii) Sample represents children from three districts which include government-aided schools from both urban and rural areas iii) Comparing WHO and IAP growth charts iii) Using growth charts for age 6-17 years iv) Comparing the charts with locality and gender.

\section{Conclusion}

Malnutrition, especially under-nutrition was always linked to a significant increase in the risk of mortality in the children. In the present study, a double burden of malnutrition prevalence is evident. A substantial proportion of the children are found to be underweight (30.6\%). Boys were found to be more severely thin than girls and girls were more overweight than the boys. Gender disparity would thrive to overnutrition and non-communicable diseases in the future if we do not act now. Rural students were more underweight whereas urban students were more overweight and obese. Malnutrition is a dual regional issue. With rapid urbanization, this burden will increase further. An agerelated decreasing trend in thinness and an increasing trend in overweight is observed. A peak increase of over-nutrition is observed among early adolescents. Both the standardized charts (WHO and IAP) had different interpretations and sensitivity in detecting under and over nutrition. The variation between two tools is $12.3 \%-24.2 \%$ for undernutrition and $0.5-1.2 \%$ for over-nutrition. The policymakers' decision depends upon these measurements and elucidation. Hence a sensitive tool designed for the Indian population to detect both under and overnutrition is required.

\section{Financial Support}

The authors have not received any financial support to conduct the research activity.

\section{Author's contributions}

Dr. Chandana Hombaiah, Dr. Anil S Bilimale, and Dr. Madhu B contributed to data collection, literature search, data analysis, data interpretation, and writing. Dr. M R Narayana Murthy contributed to data collection, data interpretation, coordination, and editing.

\section{Ethical standards disclosure}

This study was conducted according to the guidelines laid down in the Declaration of Helsinki and all procedures involving research study participants were approved by the institutional ethics committee of JSS Medical College, Mysuru. Written informed consent was obtained from all subjects and respective educational authorities.

\section{Declaration of competing interest}

None declared.

\section{Acknowledgements}

We want to thank all the faculties, Statistician, Post-graduates, Interns, Janitors, Administrative Assistants of Department of Community Medicine and School of Public Health and Principal of JSS Medical College, JSSAHER, teachers of individual JSS schools who have helped us directly or indirectly in conducting this project.

\section{References}

1 Nutrition and health in children and the role of the healthcare worker [Internet]. [cited 2019 Aug 13]. Available from: https://www.ausmed.com/cpd/articles/nutrit ion-for-children.

2 Galloway R. Global nutrition outcomes at ages 5 to 19 . In: Bundy DAP, Silva N de, Horton S, Jamison DT, Patton GC, eds. Child and Adolescent Health and Development [Internet]. third ed. Washington (DC): The International Bank for Reconstruction and Development/The World Bank; 2017 [cited 2019 Aug 13]. Available from: http: //www.ncbi.nlm.nih.gov/books/NBK525239/.

3 Aroor AR, Airody SK, Mahale R, SR R, Shetty S, Rao AR. Anthropometry and prevalence of common health problems among school going children in surathkal, Karnataka. J Clin Diagn Res. 2014. Dec;8(12): PC01-5.

$4 \mathrm{RMNCH}+\mathrm{A}$ in India.pdf [Internet]. [cited 2019 Aug 15]. Available from: https:// www.mchip.net/sites/default/files/RMNCH+A\%20in\%20India.pdf.

5 Popkin BM, Corvalan C, Grummer-Strawn LM. Dynamics of the double burden of malnutrition and the changing nutrition reality. The Lancet [Internet]. 2020 Jan [cited 2020 May 5];395(10217):65-74. Available from: https://linkinghub.elsevier. $\mathrm{com} / \mathrm{retrieve/pii/S0140673619324973.}$

6 Obesity and overweight [Internet]. [cited 2020 May 5]. Available from: https:// www.who.int/news-room/fact-sheets/detail/obesity-and-overweight.

7 Kapil U, Joshi A, Nayar D. Utility of growth monitoring: its relevance in the promotion of child health. Indian Pediatr. 1994. Feb;31(2):239-244.

8 WHO | BMI-for-age (5-19 years) [Internet]. WHO [cited 2019 Aug 14]. Available from: http://www.who.int/growthref/who2007_bmi_for_age/en/.

9 IAP growth charts [Internet]. Indian Academy of pediatrics (IAP) [cited 2019 Aug 15]. Available from: https://www.iapindia.org/iap-growth-charts/.

10 John J. Comparison of World Health Organization growth standards with Indian Academy of Pediatrics growth charts of under-five children in a rural area of Puducherry. Medical Journal of Dr. DY Patil University. 2017. Jan. 1;10(1):22.

11 Hasan I. A Study of the prevalence of malnutrition in government School children in the field area of Azad Nagar Bangalore, India. Nature Precedings. 2010. Oct. 14;1-1.

12 Rawat R, Kumar S, Jose R. Prevalence and determinants of under-nutrition among school-aged children in an urban slum in India. 2014. Nov. 20; 2:102-105.

13 Singh JP, Kariwal P, Gupta SB, Singh AK, Imtiaz D. Nutritional status and morbidity among school-going children: a scenario from a rural India. Scholars J Appl Med Sci. 2014;2(1D):379-383.

14 Epidemiology of childhood overweight \& obesity in India: a systematic review [Internet]. [cited 2020 May 2]. Available from:. https://www.ncbi.nlm.nih.gov/pmc /articles/PMC4859125/.

15 Christian Parul, Smith Emily. Adolescent undernutrition: global burden, physiology, and nutritional risks. Ann Nutr Metabol. 2018;72:316-328. https://doi.org/10.1159/ 000488865.

16 Ene-Obong H, Ibeanu V, Onuoha N, Ejekwu A. Prevalence of overweight, obesity, and thinness among urban school-aged children and adolescents in southern Nigeria. Food Nutr Bull. 2012. Dec. 1;33(4):242-250.

17 Caleyachetty R, Rudnicka AR, Echouffo-Tcheugui JB, Siegel KR, Richards N, Whincup PH. Prevalence of overweight, obesity, and thinness in 9 to 10 -year-old children in Mauritius. Glob Health. 2012. Jul. 23;8(1):28.

18 Doak CM, Adair LS, Bentley M, Monteiro C, Popkin BM. The dual burden household and the nutrition transition paradox. Int J Obes. 2005 Jan;29(1):129-136.

19 Rawat R, Garg SK, Chopra H, et al Prevalence of malnutrition among school children with reference to overweight and obesity and its associated factors. Indian $J$ Community Health. 2012. Jun. 30;24(2):97-101. 
20 Jeyaseelan L, Lakshman M. Risk factors for malnutrition in south Indian children. J Biosoc Sci. 1997. Jan;29(1):93-100.

21 Savitha MR, Kondapuram N. Comparison of 2006 WHO and Indian Academy of Pediatrics recommended growth charts of under-five Indian children. Indian Pediatr. 2012. Sep; 49(9):737-739.
22 Revised IAP growth charts for height, weight, and Body mass index for 5- to 18-yearold Indian children [Internet] [cited 2020 may 8]. Available from: https://indianped iatrics.net/jan2015/jan-47-55.htm. 